\title{
Imaging the structure of the plasma membrane with platinum replica and cryogenic electron microscopy and tomography of unroofed cells.
}

\author{
Justin Taraska and Kem Sochacki
}

NHLBI/NIH, United States

Protein complexes and organelles at the cell's surface control adhesion, membrane traffic, excitability, motility, signaling, tissue morphogenesis, and development(1). Understanding how the varied factors that drive these processes are structured in the living cell plasma membrane is essential to understanding human health and disease(2). Yet, the methods to determine the structures of native plasma membrane complexes are under-developed. We employ a method called "unroofing" to rapidly isolate large intact pieces of the plasma membrane and its associated organelles $(3,4)$. These ultra-thin membranes are optimal platforms for visualizing the structure of organelles and proteins with platinum replica transmission electron, cryogenic electron, and correlative cryogenic light and electron microcopy and tomography(2). We use this system to study the primary cellular pathway of clathrin-mediated endocytosis (CME) in mammalian cells(5). In CME, a geometric lattice of clathrin triskelia assembles at the plasma membrane to generate vesicles that curve and capture cargo into the cell(6). We first use platinum replica transmission electron microscopy and tomography to present a global structural framework of the CME pathway across dozens of cells and cell types. We determine the shape and size parameters common to clathrin-coated sites across many different systems. We show that clathrin sites maintain a constant surface area during the later stages of curvature. Next, to understand the clathrin lattice at the molecular scale, we use cryogenic electron tomography of rapidly frozen plasma membranes. We image the molecular interactions of the clathrin lattice at the scale of molecules. We find that the clathrin coat is loosely assembled and disordered in flat lattices. We propose that coalescing this sterically strained network drives the energetic transitions needed to curve a flat lattice into a vesicle. Together, these data present a new mechanistic model of clathrin mediated endocytosis at the plasma membrane. We anticipate that these imaging methods will help determine the molecular structures of other protein complexes at the plasma membrane in many cells, different tissues, and when systems are challenged with signals and disease. 
A
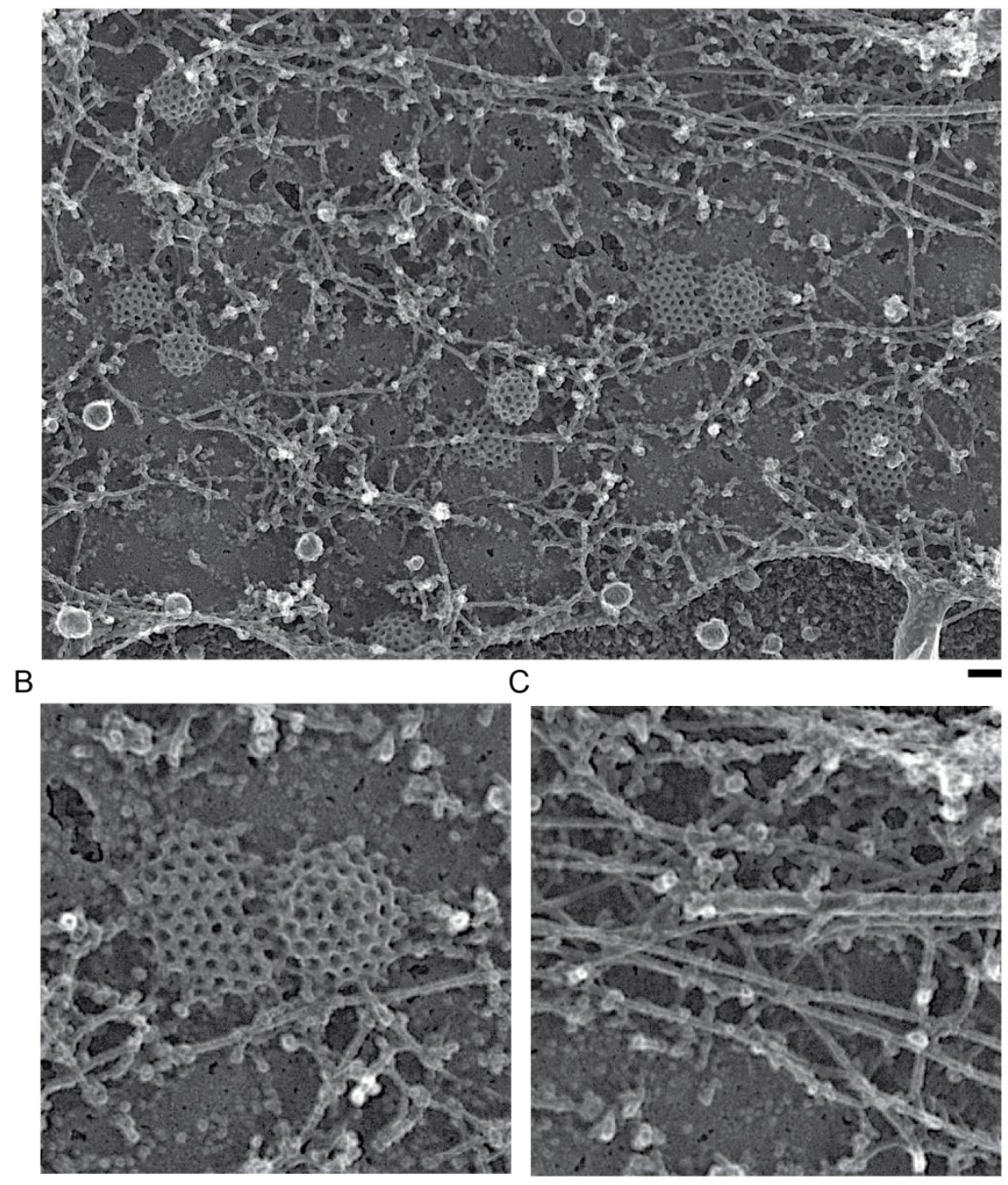

Figure 1. (A) Platinum replica transmission electron microscopy of the unroofed inner plasma membrane of a HeLa cell. (B) Clathrin coat is visible as a geometric lattice of polygons. (C) Small actin fibers and a larger microtubule form a network of filaments. Scale bar is $100 \mathrm{~nm}$.

\section{References}

1. Kusumi A, Fujiwara TK, Chadda R, Xie M, Tsunoyama TA, Kalay Z, et al. Dynamic organizing principles of the plasma membrane that regulate signal transduction: commemorating the fortieth anniversary of Singer and Nicolson's fluid-mosaic model. Annual review of cell and developmental biology. 2012;28:215-50.

2. Taraska JW. A primer on resolving the nanoscale structure of the plasma membrane with light and electron microscopy. J Gen Physiol. 2019;151(8):974-85.

3. Heuser J. The Production of 'Cell Cortices' for Light and Electron Microscopy. Traffic. 2000;1(7):54552.

4. Avery J, Ellis DJ, Lang T, Holroyd P, Riedel D, Henderson RM, et al. A cell-free system for regulated exocytosis in PC12 cells. J Cell Biol. 2000;148(2):317-24.

5. Mettlen M, Chen PH, Srinivasan S, Danuser G, Schmid SL. Regulation of Clathrin-Mediated Endocytosis. Annual review of biochemistry. 2018;87:871-96.

6. Sochacki KA, Taraska JW. From Flat to Curved Clathrin: Controlling a Plastic Ratchet. Trends Cell Biol. 2019;29(3):241-56. 